\title{
THE RELATIONSHIP OF POSTTRAUMATIC STRESS DISORDER WITH CHILDHOOD TRAUMAS, PERSONALITY CHARACTERISTICS, DEPRESSION AND ANXIETY SYMPTOMS IN PATIENTS WITH DIAGNOSIS OF MIXED ANXIETY-DEPRESSION DISORDER
}

\author{
Secil Ozen, Ercan Dalbudak \& Merve Topcu \\ Turgut Ozal University, Medicine School, Psychiatry Department, Ankara, Turkey \\ received: 12.1.2017; \\ revised: 5.12.2017; \\ accepted: 7.2 .2018
}

\begin{abstract}
SUMMARY
Background: The purpose of the current study is to investigate the relationship of symptoms of Posttraumatic Stress Disorder (PTSD) to childhood trauma and personality characteristics among patients with the diagnosis of mixed anxiety-depression disorder.

Subjects and methods: A total of 130 patients who were admitted to the psychiatry outpatient and were diagnosed with mixed anxiety-depression disorder were conveniently sampled in the current study. In order for the researchers to complete the assessment, the patients with a history of at least one traumatic experience $(40.8 \%, n=53)$ were asked to complete a socio-demographic form, the Beck Anxiety Inventory and Beck Depression Inventory, the Childhood Trauma Questionnaire-Short Form, the Posttraumatic Stress Disorder Checklist-Civilian Version, and the Eysenck Personality Questionnaire Revised/Abbreviated Form.

Results: One hundred thirty patients participated in the current study. In the study, $40.8 \%$ of the patients ( $n=53$ ) reported that they had been exposed to at least one traumatic event during their lifetime. Patients with a history of at least one traumatic experience $(40.8 \%, n=53)$ were separated into two groups: those with a diagnosis of PTSD ( $n=21)(39.6 \%)$ and those without PTSD $(n=32)(60.4 \%)$ (cut off score is 50 and above). The mean comparisons concluded that the group with PTSD and the group without PTSD were significantly different from each other in terms of depression and anxiety symptom severity, neuroticism, and history of physical abuse and neglect. Spearman's correlation analysis revealed that PTSD severity was significantly and positively correlated with neuroticism, and with depression and anxiety symptom severity, as the scores were significantly and negatively correlated with extraversion. The two-step logistic analysis first revealed the severity of the depression symptom, and secondly, physical abuse significantly predicted PTSD in the PTSD group.

Conclusions: In patients with anxiety and/or depressive disorder, PTSD is one of the most frequently-observed comorbid psychiatric disorders. The current study revealed that a history of childhood trauma, neuroticism, and introversion can be considered risk factors for PTSD among patients with mixed anxiety-depression disorder in adulthood. Therefore, it should be taken into consideration that those neurotic and introverted patients with childhood trauma history could be more at risk for PTSD.
\end{abstract}

Key words: childhood trauma - mixed anxiety-depression disorder-personality - posttraumatic stress disorder

\section{INTRODUCTION}

Patients with a diagnosis of mixed anxiety-depression disorder manifest symptoms of both depression and anxiety at the same time. However, taken separately, the symptoms of this phenomenon do not meet the diagnostic criteria (Hirschfeld 2001). While in studies differential diagnosis of anxiety and depression cannot be met in patients with mild manifestations, some of these patients are diagnosed mixed anxietydepression (Angst 1997).

It is predicted that at least $50 \%$ of adults experience a traumatic event during their lifetime (Kessler et al. 1995). Five-ten percent (5-10\%) of those who have experienced a traumatic event develop posttraumatic stress disorder (PTSD). Other psychiatric disorders are observed to be comorbid with PTSD as well. It was revealed that $88 \%$ of those with a diagnosis of PTSD also have comorbid depression, anxiety, and abuse of at least one substance. Also, $59 \%$ of them have two diag- noses comorbid with PTSD (Kessler et al. 1995, Ginzburg et al. 2010). It was determined that PTSD has a strong relationship with symptoms of anxiety and depression (Campell et al. 2015).

Although traumatic events are an apriori condition for the development of PTSD, it is known that not every individual who experiences trauma develops PTSD. The experience of a traumatic event is not the only criterion. It is suggested that it is essential to examine the psychological meaning and effect of the event. One vital factor regarding the psychological meaning of the event is that the patient experienced the trauma in the early years of life (Burgess et al. 1995). Also, the type of events, environmental factors, the severity of the clinical manifestations of the trauma, personality dispositions, sociodemographics, and previous history of mental health are found to be influential risk factors for the development of PTSD (Breslau et al. 1998). Higher neuroticism, introversion (Resnick et al. 1989, Breuslau \& Davis 1992), previous history of psychiatric disorders such as 
anxiety or major depressive disorders (Ozgen \& Aydin 1999), psychological meaning of the event, alcohol use, manifestations of borderline, paranoid, antisocial or dependent personality features, trauma exposure in early years of life (Breslau et al. 1998) are factors increasing the likelihood and severity of PTSD.

Regarding childhood trauma, it was suggested that individuals develop behavioral coping strategies which are specifically calibrated to deal with childhood trauma (e.g., quick reaction, despair, avoidance, disruptive behaviors, etc.). These coping behaviors are transmitted into adulthood through cognitive schemata. It was thought that children who have been exposed to traumatic events perpetuate the early experience of their trauma. As adults, they may exhibit violent and abusive behavior, perpetuating the traumatic events of their past within relationships developed in adulthood (Burgess et al. 1995). It is known that exposure to early childhood trauma is found to be related to a number of psychiatric disorders, and it is accepted as a risk factor for the development of psychiatric disorders (Bryer et al. 1987). Studies have revealed that a history of childhood trauma is associated with PTSD in adulthood (McCutcheon et al. 2010), with personality disorders (Haller \& Miles 2004), and with psychiatric disorders such as substance abuse (Toker et al. 2011). It has been found that $11 \%$ of adult patients with PTSD experienced childhood trauma (Eilenberg et al. 1996). There has been specific emphasis on the relationship between a history of childhood trauma with anxiety and depression (Ozgen \& Aydin 1999, Hovens et al. 2010, Orsel et al. 2011).

Personality characteristics are one of the most important risk factors in the development of PTSD (Resnick 1989, Breuslau \& Davis 1992). It was revealed that, as personality characteristics have an influential role in determining reactions in the aftermath of traumatic events (Campbell et al. 2015, Miller et al. 2012, Wolf et al. 2012), traumatic events also have an influence on changing personality characteristics (Widiger 2011). It was suggested that neuroticism dimension of personality points out over responsiveness; and individuals with higher neuroticism could also be anxious, depressed, introverted and have low self-esteem (Eysenck \& Eysenck 1985). Neuroticism is considered to be influential in the development of psychological problems (Ormal \& Wohlfarth 1991) and anxiety disorders (Clark et al. 1994). While neuroticism may increase the sensitivity to PTSD, repeated exposure to traumatic events may increase neuroticism (Eberly et al. 1991). It has been reported that there is a positive correlation between PTSD and neuroticism (Brodaty et al. 2004). A previous history of psychiatric disorders and neuroticism was found to be more influential in the development of PTSD than the severity of the stress caused by the traumatic event (McFarlane 1989). When the degree of extraversion is low, there is found to be a strong relationship between anxiety and PTSD (Campell et al. 2015). In patients who have PTSD comorbid with anxiety disorders and depression, a higher neuroticism and lower extraversion were found (Miller et al. 2012, Wolf et al. 2012).

PTSD is frequently seen as a comorbid disorder with depression and anxiety disorders. In the literature so far, there has not been any study which examines the prevalence of PTSD in patients with a history of childhood trauma, and the relationship of PTSD with childhood traumas and personality types among patients with mixed anxiety-depression disorder. Mixed anxietydepression is a disorder in which the manifestation of depressive and anxiety symptoms are observed together, but these symptoms observed separately do not meet the criteria for an individual diagnosis of depression, or an individual diagnosis of anxiety. Therefore, exploring traumatic experiences and comorbid PTSD related to early experiences could be essential in patients without an individual diagnosis of anxiety disorders or depression. The aim of the current study is to examine the relationship of PTSD symptoms to childhood trauma, to personality characteristics, and to depressive and anxiety symptoms in patients with the diagnosis of mixed anxiety-depression disorder. It is hypothesized that PTSD will be significantly predicted by childhood trauma, personality characteristics, and depressive and anxiety symptoms.

\section{SUBJECTS AND METHODS}

Patients, who sought treatment at the psychiatry clinic at Turgut Ozal University during the period of July 2014 to February 2015 were interviewed by a psychiatrist using the ICD-10 criteria. Of these patients, 178 of them were diagnosed with mixed anxiety-depression disorder. These patients were orally informed about the current study and 130 patients volunteered to participate. The clinician excluded 48 patients who did not agree to participate in the study. Written informed consent forms were obtained from the patients who agreed to participate in the study. After the researchers conducted clinical interviews with the patients based on the ICD-10, the patients without a diagnosis of mixed anxiety-depression disorder, and those with depression, anxiety disorders (e.g., generalized anxiety disorder, social phobia, obsessive-compulsive disorder, panic disorder, etc.), somatoform, psychotic and bipolar disorders were excluded by the clinician. Initially, the patients with a diagnosis of mixed anxiety-depression disorder were asked one question about their previous exposure to traumatic events: "When individuals experience, witness, or are confronted with serious injury or death threats, or threats to their physical integrity or to the physical integrity of others, people may face serious problems. Have you ever experienced, witnessed, or been confronted with at least one of these types of events during your lifetime?" The number of patients who answered this question as "YES" was 53. These patients, who reported a history of traumatic events, completed the socio- 
demographic form, the Beck Depression Inventory, the Beck Anxiety Inventory, the PTSD Checklist-Civilian Version, the Eysenck Personality Questionnaire Revised/Abbreviated Form, and the Childhood Trauma Questionnaire. The patients were informed about the study and their consent was obtained. Ethical approval was received from the University's ethics committee.

\section{Instruments}

Sociodemographic form: This form includes questions examining the participant's age, gender, educational status, previous psychiatric history, and history of trauma.

Beck Depression Inventory (BDI): This scale was originally developed by Beck (1961). It is a self-report measure comprising 21 items that are rated in a range between $0-63$. Studies on the scale's reliability and validity in the Turkish culture were conducted by Hisli (1989).

Beck Anxiety Inventory (BAI): This scale was originally developed by Beck et al. (1988). It is a self-report measure comprising 21 items which are rated in a range between 0-63. Studies on the scale's reliability and validity in the Turkish culture were conducted by Ulusoy et al. (1998).

Posttraumatic Stress Disorder Checklist-Civilian Version $(P C L-C)$ : This scale was originally developed by Weathers et al. (1993). It contains 17 items and a 4point-likert-type scale that measures all symptoms of PTSD as defined in the DSM-IV. A total score above 50 reveals a diagnosis of PTSD. The scale was adapted to the Turkish culture by Kocabasoglu et al. (2005).

Eysenck Personality Questionnaire Revised/Abbreviated Form (EPQR-A): The initial form was comprised of 48 items, but the scale was revised, and the revised form includes only 24 items (Francis et al. 1992). The scale has three dimensions that measure personality, namely, extraversion, neuroticism and psychoticism. The Lie subscale was developed for control. Each factor has 6 items. The scale was adapted to the Turkish culture by Karanc1 et al. (2007). Good reliability and validity statistics were reported as well (Karanc1 et al. 2007).

Childhood Trauma Questionnaire-Short Form (CTQ28): This scale was originally developed by Bernstein in 1994 as a 70-item self-report measure. Later, Bernstein revised the scale and it was shortened to include only 28 items (Bernstein et al. 1994, Bernstein 2003). The scale has 5 factors which examine 5 types of childhood trauma, namely, abuse and neglect, physical abuse, emotional abuse, sexual abuse, and neglect. The scale was adapted to the Turkish culture by Sar et al. (2012).

\section{Statistical Analyses}

All statistical analyses were completed using the IBM SPSS 20.0. Group means, standard deviations and frequencies with percentages were given. Spearman's correlation coefficients were calculated in order to determine the relationship of PTSD to anxiety and depressive symptom severity, to the Eysenck perso- nality dimensions, and to childhood trauma in patients with a previous traumatic event history. The patients with a previous history of trauma were separated into two groups, namely, those with PTSD and those without PTSD. The means of the group with PTSD and the means of the group without PTSD were compared using the Mann-Whitney U test. A logistic regression analyses was completed in order to measure if the severity of the depressive and anxiety symptoms, the personality characteristics, and the childhood trauma could predict PTSD. This was completed in both the group with PTSD, and the group without PTSD. The significance level of the test was accepted as $\mathrm{p}<0.05$.

\section{RESULTS}

One hundred thirty patients participated in the current study. Of these, $49.2 \%$ of them were female $(n=64)$, and $50.8 \%$ of them were male $(n=66)$. The mean age of the participants was 27.39 with a standard deviation of \pm 10.56 . In the study, $40.8 \%$ of the patients $(n=53)$ reported that they had been exposed to at least one traumatic event during their lifetime. Of those with a previous history of trauma, $50.9 \%$ were female $(n=27)$ and $49.1 \%$ were male $(n=26)$. The mean age was 29.60 with a standard deviation of \pm 10.44 .

In patients with a previous history of at least one traumatic event $(n=53)$, the PTSD severity had a significantly high positive correlation with depressive symptom severity $(\mathrm{r}=0.74, \mathrm{p}<0.01)$; a positive correlation with anxiety symptom severity $(\mathrm{r}=0.49, \mathrm{p}<0.01)$ and a neuroticism subscale of Eysenck personality dimensions $(\mathrm{r}=0.34, \mathrm{p}<0.05)$. The PTSD severity had a significantly negative correlation with the extraversion subscale of Eysenck personality dimensions $(\mathrm{r}=-0.38$, $\mathrm{p}<0.01$ ) (Table 1).

The mean scores of the severity of the depressive and anxiety symptoms, the Eysenck personality dimensions and the childhood trauma in patients with a previous traumatic event history are summarized in Table 2. Patients with a previous history of traumatic events were separated into two groups, those with a diagnosis of PTSD and those without PTSD (cut off score is 50 and above). While $39.6 \%$ of these patients $(n=21)$ were in the PTSD group, $60.4 \%$ of the patients $(n=32)$ were in the group without PTSD. Of those who were in the group with PTSD, $47.6 \%$ are female $(n=10)$, and $52.4 \%$ are male $(n=11)$. The mean age of the patients with a previous history of traumatic events was found to be 28.14 with a standard deviation of \pm 11.63 .

In the group with PTSD $(n=21)$, the frequency distribution across gender was insignificant $\left(\chi^{2}=0.05\right.$, $\mathrm{p}=0.827)$. The means scores of those in the group with PTSD and the mean scores of those in the group without PTSD were compared across depressive and anxiety symptom severity, the Eysenck personality dimensions, and childhood traumas. Results of the Mann-Whitney U test revealed that the two groups were significantly 
different from each other in terms of neuroticism $(Z=-2.31, p<0.05)$, anxiety $(Z=-2.97, p<0.01)$ and depressive $(Z=-4.93, p<0.01)$ symptom severity, physical neglect $(Z=-1.97, p<0.05)$ and physical abuse $(Z=-2.25$, $\mathrm{p}<0.05$ ) (Table 2).

A logistic regression analysis was completed in order to measure the predictive capacity regarding PTSD of depressive and anxiety symptom severity, personality characteristics and childhood trauma, in both the group with PTSD and without PTSD. In the first step, the depressive and anxiety symptom severity was entered into the equation as control variables. The model was significant for the first step $\left(\chi^{2}=30.08\right.$, $\mathrm{p}<0.001)$. However, only the depressive symptom severity was a significant predictor in the PTSD group $(\beta=0.16$, Wald $Z=13.06, \operatorname{Exp}(B)=1.17, p<0.001)$. In the second step, the Eysenck personality dimensions and the reports of childhood traumas were entered into the equation. The model was still found to be significant $\left(\chi^{2}=44,09, p<0.001\right)$. Although the Eysenck personality dimensions and the reports of childhood traumas were added into the equation, the depressive symptom severity was still a significant predictor of the PTSD group $(\beta=0.22$, Wald $Z=7.62, \operatorname{Exp}(B)=1.24, p=0.006)$. Besides depressive symptom severity, only physical abuse was a significant predictor in the PTSD group $(\beta=1.04$, Wald $Z=5.36$, Exp $(B)=2.83, p=0.021)$. On the other hand, a variation of anxiety symptom severity was found to be at a statistical threshold in the prediction of PTSD in the PTSD group ( $\mathrm{p}=0.046)$ (Table 3).

Table 1. According to Spearman's correlation analysis, relationship of PTSD symptom severity with depressive and anxiety symptom severity, childhood traumas, and personality characteristics

PLC-C BDI BAI $\begin{gathered}\text { Neuro- Extra- Psycho- } \\ \text { ticism version ticism }\end{gathered}$ Lie $\begin{gathered}\text { Emo- } \\ \text { tional Physical } \\ \text { neglect }\end{gathered}$ neglect $\begin{gathered}\text { Emo- } \\ \text { tional Physical Sexual } \\ \text { abuse abuse abuse }\end{gathered}$

\begin{tabular}{lccccccccccccc}
\hline BDI & $0.74^{* *}$ & 1 & & & & & & & & & \\
BAI & $0.49^{* *}$ & $0.50^{* *}$ & 1 & & & & & & & & \\
Neuroticism & $0.34^{*}$ & $0.37^{* *}$ & 0.12 & 1 & & & & & & \\
Extraversion & $-0.38^{* *}$ & $-0.27^{*}$ & -0.15 & $-0.43^{* *}$ & 1 & & & & & & & \\
Psychoticism & 0.01 & -0.04 & -0.15 & -0.22 & $0.29^{*}$ & 1 & & & & & \\
Lie & -0.24 & -0.23 & -0.06 & -0.07 & 0.03 & $-0.35^{* *}$ & 1 & & & & \\
Emotional neglect & -0.28 & -0.12 & $-0.35^{*}$ & -0.19 & 0.25 & 0.12 & -0.06 & 1 & & & \\
Physical neglect & 0.23 & 0.22 & 0.16 & $0.35^{*}$ & -0.19 & -0.06 & -0.07 & $-0.42^{* *}$ & 1 & & \\
Emotional abuse & -0.25 & -0.22 & -0.14 & $-0.37^{* *}$ & $0.31^{*}$ & -0.03 & 0.04 & 0.24 & $-0.47^{* *}$ & 1 & \\
Physical abuse & 0.20 & 0.13 & 0.00 & 0.00 & -0.07 & -0.07 & -0.14 & -0.24 & $0.29^{*}$ & -0.01 & 1 \\
Sexual abuse & -0.24 & -0.25 & -0.19 & $-0.46^{* *}$ & 0.19 & -0.03 & 0.06 & $0.28^{*}$ & $-0.57^{* *}$ & $0.74^{* *}$ & -0.16 & 1 \\
CTQ-28 Total & -0.07 & -0.13 & -0.21 & $-0.36^{* *}$ & 0.25 & 0.02 & -0.11 & 0.24 & -0.16 & $0.80^{* *}$ & $0.32^{*}$ & $0.71^{* *}$ \\
\hline
\end{tabular}

PTSD: Posttraumatic Stress Disorder; PCL-C: Posttraumatic Stress Disorder Checklist-Civilian Version; BDI: Beck Depression Inventory; BAI: Beck Anxiety Inventory; CTQ-28: Childhood Trauma Questionnaire; $* \mathrm{p}<0.05 ; * * \mathrm{p}<0.01$

Table 2. Mean comparisons of PTSD and without PTSD groups across depressive and anxiety symptom severity, childhood traumas, and Eysenck personality characteristics

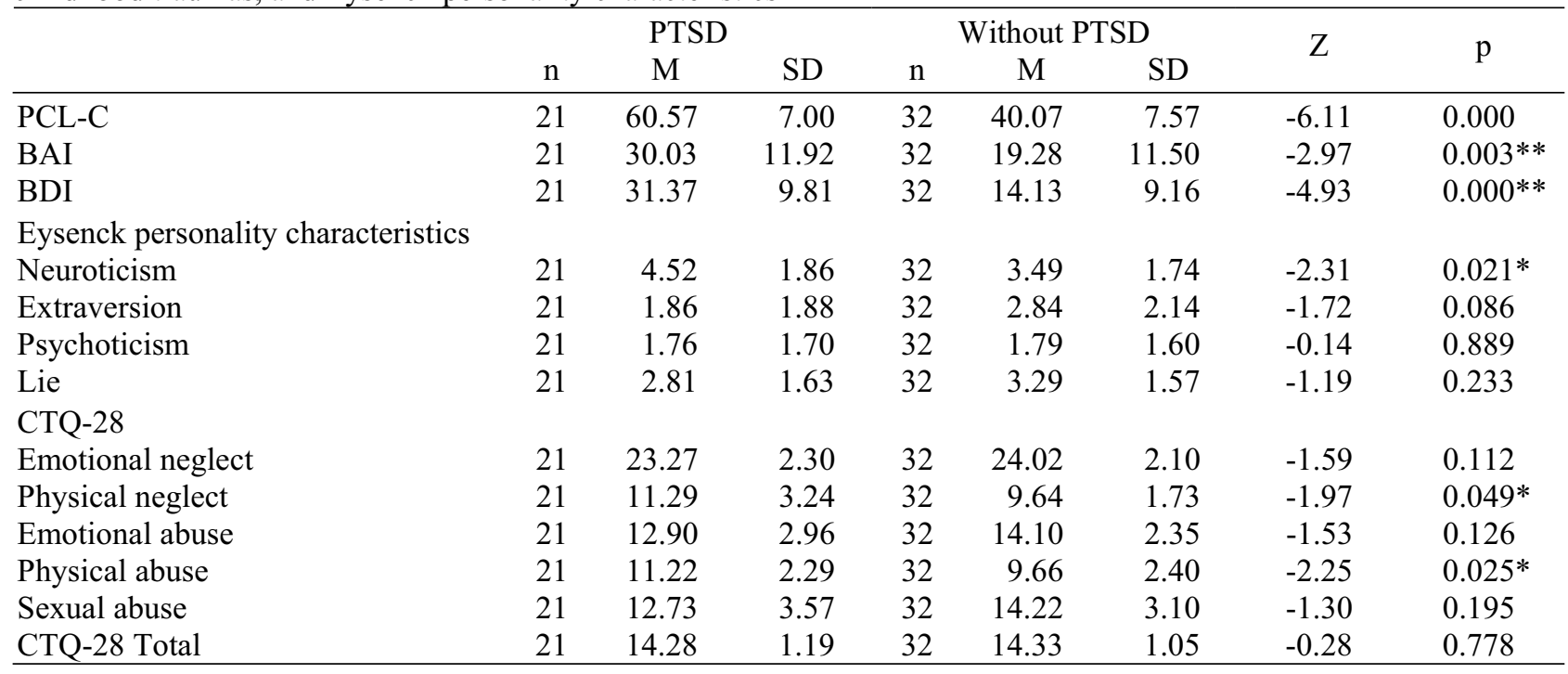

PTSD: Posttraumatic stress disorder; PCL-C: Posttraumatic Stress Disorder Checklist-Civilian Version; BAI: Beck Anxiety Inventory; BDI: Beck Depression Inventory; CTQ-28: Childhood Trauma Questionnaire; ${ }^{*} \mathrm{p}<0.05 ; * * \mathrm{p}<0.01$ 
Table 3. According to logistic regression analysis, diagnosis of PTSD is predicted variables

\begin{tabular}{lcccccc}
\hline & $\mathrm{B}$ & $\mathrm{SE}$ & Wald Z & $\mathrm{p}$ & $\operatorname{Exp}(\mathrm{B})$ & $\mathrm{CI}$ \\
\hline First step & & & & & & \\
BAI & 0.04 & 0.04 & 1.38 & 0.241 & 1.05 & $0.97-1.12$ \\
BDI & 0.16 & 0.04 & 13.06 & $<0.001$ & 1.17 & $1.07-1.27$ \\
Second step & & & & & & \\
BAI & 0.17 & 0.08 & 4.16 & 0.046 & 1.19 & $1.01-1.40$ \\
BDI & 0.22 & 0.08 & 7.62 & 0.006 & 1.24 & $1.06-1.44$ \\
Neuroticism & 0.60 & 0.42 & 2.06 & 0.151 & 1.82 & $0.80-4.12$ \\
Extraversion & -0.06 & 0.30 & 0.04 & 0.836 & 0.94 & $0.52-1.70$ \\
Psychoticism & 0.25 & 0.42 & 0.37 & 0.542 & 1.29 & $0.57-2.92$ \\
Lie & 0.55 & 0.40 & 1.86 & 0.173 & 1.73 & $0.79-3.78$ \\
Emotional neglect & 0.63 & 0.39 & 2.60 & 0.107 & 1.88 & $0.87-4.06$ \\
Physical neglect & 0.67 & 0.42 & 2.48 & 0.107 & 1.95 & $0.85-4.48$ \\
Emotional abuse & -0.83 & 0.45 & 3.46 & 0.063 & 0.44 & $0.18-1.05$ \\
Physical abuse & 1.04 & 0.45 & 5.36 & 0.021 & 2.83 & $1.17-6.80$ \\
Sexual abuse & 1.00 & 0.56 & 3.15 & 0.076 & 2.72 & $0.90-8.19$ \\
\hline PTSD: Post
\end{tabular}

PTSD: Posttraumatic Stress Disorder; CI: Critical interval; BAI: Beck Anxiety Inventory; BDI:Beck: Depression Inventory

\section{DISCUSSION}

It has been suggested that traumatic events may make individuals prone to psychiatric disorders (Garmezy 1985). It is assumed that a high rate of exposure to traumatic events is prevalent within the general population (Kessler et al. 1995). In the current study, $40.8 \%$ of the patients with a diagnosis of mixed anxiety-depression disorder reported exposure to at least one traumatic event during their lifetime.

Studies have reported that anxiety, depression and substance abuse are frequently comorbid with PTSD (Kessler et al. 1995, Ginzburg 2010). A higher neuroticism and lower extraversion were found in patients with PTSD, and in patients with comorbid disorders such as depression and anxiety (Miller et al. 2012, Wolf et al. 2012, Kotov et al. 2010). In the current study, when the researchers compared the relationship of PTSD symptoms to depression and anxiety symptom severity, to personality characteristics and to childhood traumas, among those who reported a previous history of traumatic events, it was found that PTSD symptom severity has a significantly positive correlation with neuroticism, and a negative correlation with extraversion. These results are in accord with previous findings. In accordance with the study's hypothesis, it was found that those in the PTSD group had higher mean scores regarding depression and anxiety symptom severity, neuroticism, and physical abuse and neglect, than those in the group without PTSD.

The prevalence of the rate of depression, which is frequently comorbid with PTSD, is $49 \%$ (Kessler et al. 1995). In accord with previous findings, it was found that the PTSD group had higher scores regarding the severity of symptoms of depression. While depression could be a result of prolonged PTSD, a previous history of depression may lead to the development of PTSD. Depression and PTSD could be individual disorders which patients could develop during the aftermath of exposure to a traumatic event. The neurobiological basis of both disorders are claimed to be common (Brady et al. 2000).

Anxiety is rooted in a tendency to perceive a stressful situation as a threat. In individuals with a higher level of anxiety, the autonomic nervous system overreacts in the case of traumatic events (Marais \& Stuart 2006). A study on anxiety and PTSD symptoms following a traumatic event revealed that anxiety is a precursor for PTSD (Collimore et al. 2008). One study, which was carried out in order to examine the relationship among anxiety, PTSD symptoms and somatic complaints after a hurricane, suggested a strong association between anxiety and PTSD symptoms. It was found that anxiety is a unique predictor of PTSD and somatic complaints (Hensley \& Varela 2008). In accord with previous findings in the literature, it was found in the current study that those with a diagnosis of PTSD had higher scores on anxiety. It can be concluded that a higher prevalence of PTSD, comorbid with depression and anxiety, influenced the anxiety scores in the group with PTSD.

PTSD is commonly seen in adults who were physically and/or sexually abused in childhood (Kessler et al. 1995). One study which was conducted with veterans, who had been diagnosed with PTSD, and who had received psychiatric treatment, revealed that $26 \%$ of patients with PTSD, and 7\% of controls, reported physical abuse in childhood (Bremner et al. 1993). Similarly, the rate of exposure to physical abuse and neglect was significantly higher in the PTSD group, than in the group without PTSD in the current study. Considering these findings, childhood trauma could be associated with manifestations of PTSD in adulthood. Therefore, it could be speculated that physical abuse and neglect in childhood may increase the likelihood of PTSD after exposure to traumatic events later in life. 
One of the essential risk factors in the development of PTSD is personality characteristics (Resnick et al. 1989, Breuslau \& Davis 1992). As a dimension of personality, neuroticism is a vital precursor for future mood and eating disorders, anxiety, PTSD and substance abuse, as a reaction to lifelong stress (Malouff et al. 2005). Bramsen and van der Ploeg (1999) suggested that those who had been exposed to traumatic experiences have higher scores on anxiety, depression, and aggression, than those who had not experienced trauma. Additionally, it was claimed that stress during war may alter some personality characteristics. Brodaty et al. (2004) reported that neuroticism alone is associated with PTSD. In another study, examining PTSD after a snowstorm in China, it was determined that neuroticism and negative coping are influential (Wu et al. 2011). In the current study, neuroticism and PTSD symptom severity are positively correlated with each other. Also, it was found that neuroticism scores were higher in the group with PTSD, than in the group without PTSD. In this regard, it was considered that cognitive and affective components of the personality, including expectations, beliefs, information coding, coping strategies, etc., may increase the risk of developing PTSD. Neuroticism in particular, when present with depressive and anxiety symptoms, may become crucial in determining PTSD symptom severity.

Lastly, it was found that, in addition to depressive symptoms, physical abuse was a significant predictor within the PTSD group. It was thought that physical abuse in childhood may become a risk factor for depression, and for developing PTSD later in life. Green et al. (2010) reported that physical and sexual abuse were found to be risk factors for PTSD. Only physical abuse was found to be a risk factor in the current study, while sexual abuse was insignificant. In the current study, a history of childhood abuse and neglect was documented through self-reports, and this was done retrospectively. Therefore, it can be said that the data which was gathered through self-reports and retrospectively, may yield a bias in reporting the actual rates of the prevalence of abuse and neglect. Future studies with a larger sample size, including patients and control groups, are required.

Studies have revealed that a previous psychiatric history, and neuroticism, are predictors of PTSD, more so than the level of stress that was experienced during the event (McFarlane 1989, Wu et al. 2011). Neuroticism and introversion were significantly correlated with PTSD symptom severity, but they were not significant predictors in the PTSD group in the current study. Neuroticism is closely associated with depression (Eysenck \& Eysenck 1985). The reason why neuroticism was insignificant may be that depressive symptoms have more predictive capacity for PTSD.

Trauma is a risk factor not only for PTSD, but also for depression and anxiety disorders. When considering the results of the current study, it can be said that patients with a diagnosis of PTSD reported more physical abuse and neglect in childhood, had more severe depressive and anxiety symptoms, and manifested more neurotic and introvert personality characteristics. Patients who seek treatment at general psychiatry clinics because of depression and anxiety symptoms should be examined in terms of exposure to traumatic events, and for possible PTSD. Although a history of childhood trauma, introversion, and neuroticism increase the likelihood of PTSD, depressive symptom severity is the essential predictor of PTSD. Therefore, examining the history of traumatic events and exploring childhood trauma in patients with depression during the daily clinical practice may be helpful in the prediction of PTSD.

The limitations of the current study include: the small sample size, having a single question that covers previous traumatic events, using self-report measures even though the patients with a diagnosis were included in the study by means of a clinical interview, and the diagnosis of PTSD through a self-report measure. The number of traumas in the individual included in the study and the lack of indication of the duration of the anxiety depression diagnosis and the beginning of the traumatic was limitations. Also, another limitation in the current study was the lack of a control group. The fact that mixed anxiety-depression disorder and PTSD had similar symptoms is one of the limitations of this study. Despite these limitations, the current study can be considered a pilot study which examines the frequency of PTSD and its relationship with childhood trauma and personality characteristics among patients with the diagnosis of mixed anxiety and depression disorder, in which symptoms considered separately do not meet an individual diagnostic criteria. Further studies based on clinical interviews and with a larger sample size would be informative.

\section{Acknowledgements: None.}

Conflict of interest: None to declare.

\section{Contribution of individual authors:}

Secil Ozen: study design, literature review, first draft , writing the manuscript, approval of the final version;

Ercan Dalbudak: study design, first draft, approval of the final version;

Merve Topcu: statistical analyses, approval of the final version.

\section{References}

1. Angst J: Depression and anxiety: Implications for nosology, course, and treatment. J Clin Psychiatry 1997; 58:3-5

2. Beck AT: An inventory for measuring depression. Arch Gen Psychiatry 1961; 4:561-71 
3. Beck AT, Epstein N, Brown G \& Steer RA: An inventory for measuring clinical anxiety: Psychometric properties. $J$ Consult Clin Psychol 1988; 56:893-97

4. Bernstein DP, Fink L, Handelsman L, Foote L, Lovejoy M, Wenzel $K$ et al.: Initial reliability and validity of a new retrospective measure of child abuse and neglect. Am J Psychiatry 1994; 151:1132-36

5. Bernstein DP, Stein JA, Newcomb MD, Walker E, Pogge $D$, Ahluvalia $T$ et al.: Development and validation of a brief screening version of the Childhood Trauma Questionnaire. Child Abuse Negl 2003; 27:169-90

6. Brady KT, Killeen TK, Brewerton $T \&$ Lucerini $S$ : Comorbidity of psychiatric disorders and posttraumatic stress disorder. J Clin Psychiatry 2000; 61: 22-23

7. Bramsen I \& van der Ploeg HM: Fifty years later: the long-term psychological adjustment of ageing World War II survivors. Acta Psychiatr Scand 1999; 100: 350-58

8. Bremner JD, Southwick SM, Johnson DR, Yehuda $R \&$ Charney DS: Childhood physical abuse and combatrelated posttraumatic stress disorder in Vietnam veterans. Am J Psychiatry 1993; 150:235-39

9. Breslau $N \&$ Davis GC: Posttraumatic stress disorder in an urban population of young adults: risk factors for chronicity. Am J Psychiatry 1992; 149:671-75

10. Breslau N, Kessler RC, Chilcoat HD, Schultz LR, Davis $G C$ \& Andreski P: Trauma and posttraumatic stress disorder in the community: the 1996 Detroit Area Survey of Trauma. Arch Gen Psychiatry 1998; 55:626-32

11. Brodaty $H$, Joffe $C$, Luscombe $G$ \& Thompson C: Vulnerability to post-traumatic stress disorder and psychological morbidity in aged holocaust survivors. Int $J$ Geriatr Psychiatry 2004; 19:968-79

12. Bryer JB, Nelson BA, Miller JB \& Krol PA: Child-hood sexual and physical abuse as factors in adult psychiatric illness. Am J Psychiatry 1987; 144:1426-30.

13. Burgess AW, Hartman CR \& Clements PT: Biology of memory and childhood trauma. J Psychosoc Nurs Ment Health Serv 1995; 33:16-26

14. Campbell SB, Renshaw KD \& Righter JB: The role of personality traits and profiles in posttrauma comorbidity. J Trauma Dissociation 2015; 16:197-210

15. Clark L, Watson D \& Mineka S: Temperament, personality and the mood and anxiety disorders. J Abnorm Psychol 1994;103:103-16

16. Collimore KC, McCabe RE, Carleton RN \& Asmundson GJG: Media exposure and dimensions of anxiety sensitivity: differential associations with PTSD symptom clusters. J Anx Disord 2008; 22:1021-28

17. Eberly RE, Harkness AR \& Engdahl BE: An adaptational view of trauma response as illustrated by the prisoner of war experience. J Trauma Stress 1991;4:363-80

18. Eilenberg J, Fullilove MT, Goldman $R G$ \& Mellman L: Quality and use of trauma histories obtained from psychiatric outpatients through mandated inquiry. Psychiatr Serv 1996; 47:165-69

19. Eysenck $H J$ \& Eysenck $M W$ : Personality and Individual Differences: A Natural Science Approach. New York: Plenum, 1985

20. Francis LJ, Brown LB \& Philipchalk R: The development of an abbreviated form of the Revised Eysenck Personality Questionnaire (EPQR-A): its use among students in England, Canada, the USA and Australia. Pers Individ Dif, 1992; 13:443-49
21. Garmezy N: Stress-resistant children; the search for protective factors. J Child Psych Adolesc Psychiatry 1985; 4:213-33

22. Ginzburg K, Ein-Dor T \& Solomon Z: Comorbidity of posttraumatic stress disorder, anxiety, and depression: A 20-year longitudinal study of war veterans. J Affect Disord 2010; 123:249-57

23. Green JG, McLaughlin KA, Berglund PA, Gruber MJ, Sampson NA, Zaslavsky AM et al.: Childhood adversities and adult psychopathology in the National Comorbidity Survey Replication (NCS-R) I: Associations with first onset of DSM-IV disorders. Arch Gen Psychiatry 2010; 67:113-23

24. Haller DL \& Miles DR: Personality distubances in drugdependent women: Relationship to childhood abuse. Am J Drug Alcohol Abuse 2004; 30:269-86

25. Hensley L \& Varela RE: PTSD symptoms and somatic complaints following Hurricane Katrina: the roles of trait anxiety and anxiety sensitivity. J Clin Child Adolesc Psychol 2008; 37:542-52

26. Hirschfeld RM: The comorbidity of major depression and anxiety disorders: Recognition and management in primary care. Prim Care Companion J Clin Psychiatry 2001; 3:244-54

27. Hisli $N:$ Reability and validity of beck depression inventory among university students. Turkish Journal of Psychology 1989; 7:3-13. (Turkish)

28. Hovens JG, Wiersma JE, Giltay EJ, van Oppen $P$, Spinhoven P, Penninx BW et al.: Childhood life events and childhood trauma in adult patients with depressive, anxiety and comorbid disorders vs. controls. Acta Psychiatr Scand 2010; 122:66-74

29. Karancı AN, Dirik $G$ ve Yorulmaz O: Reliability and Validity Studies of Turkish Translation of Eysenck Personality Questionnaire Revised-Abbreviated. Turkish J Psychiatry 2007; 18:254-261

30. Kessler RC, Sonnega A, Bromet E, Hughes $M \&$ Nelson $C B$ : Posttraumatic stress disorder in the National Comorbidity Survey. Arch Gen Psychiatry 1995; 52:1048-60

31. Kocabasoglu N, Corapcioglu Ozdemir A, Yarglc I \& Geyran P: The Validity and safety of Turkish "PTSD Checkust-Civilian Version" (PCL-C) Scale. New Symposium 2005; 43:126-34

32. Kotov R, Gamez W, Schmidt F\& Watson D: Linking "big" personality traits to anxiety, depressive, and substance use disorders: A meta-analysis. Psychol Bull 2010; 136: 768-821

33. Malouff JM, Thorsteinsson EB \& Schutte NS: The relationship between the five-factor model of personality and symptoms of clinical disorders: a meta-analysis. $J$ Psychopathol Behav 2005; 27:101-14

34. Marais $A \&$ Stuart AD: The role of temperament in the development of Post-traumatic Stress Disorder amongst journalists. S Afr J Psychol 2006; 35:89-105

35. McCutcheon VV, Sartor CE, Pommer NE, Bucholz KK, Nelson EC, Madden PA et al.: Age at trauma exposure and PTSD risk in young adult women. J Trauma Stres 2010; 23:811-14

36. McFarlane AC: The aetiology of post-traumatic morbidity: predisposing, precipitating and perpetuating factors. Br J Psychiatry 1989;154:221-28

37. Miller $M W$, Wolf EJ, Reardon A, Greene A, Ofrat $S \&$ McInerney S: Personality and the latent structure of PTSD comorbidity. J Anxiety Disord 2012; 26:599-607 
38. Ormel $J \&$ Wohlfarth T: How neuroticism, long-term difficulties, and life situation change influence psychological distress: a longitudinal model. J Pers Soc Psychol 1991; 60:744-55

39. Orsel S, Karadag H, Kahilogulları AK \& Aktas EA. The frequency of childhood trauma and relationship with psychopathology in psychiatric patients. Anatolian $J$ Psychiatry 2011; 12:130-36

40. Ozgen F ve Aydin H: Posttraumatic Stress Disorder. Klinik Psikiyatri 1999; 1:34-41. (Turkish)

41. Resnick HS, Foy DW, Donahoe CP \& Miller EN: Antisocial behavior and posttraumatic stress disorder in Vietnam veterans. J Clin Psychol 1989; 45:860-66

42. Sar V, Ozturk E \& Ikikardes E: Validity and Reliability of the Turkish Version of Childhood Trauma Questionnaire. Turkiye Klinikleri J Med Sci 2012;32:1054-63

43. Toker T, Tiryaki A, Ozcurumez G \& Iskender B. The relationship between traumatic childhood experiences and proclivities towards substance abuse, self-esteem and coping strategies. Turk Psikiyatri Derg 2011; 22:83-92
44. Ulusoy M, Sahin NH \& Erkmen H: Turkish version of the Beck Anxiety Inventory: Psychometric properties. J Cogn Psychother 1998;12:163-72

45. Weathers FW, Litz BT, Herman DS, Jusca J \& Keane TM: The PTSD Checklist: Reliability, validity and diagnostic utility. Paper presented at the annual meeting of the International Society for Traumatic Stress Studies. San Antonio: Texas, 1993

46. Widiger TA: Personality and psychopathology. World Psychiatry 2011; 10:103-6

47. Wolf EJ, Miller MW, Harrington KM \& Reardon A: Personality based latent classes of posttraumatic psychopathology: Personality disorders and the internalizing/externalizing model. J Abnorm Psychol 2012; 121:256-62

48. Wu D, Yin H, Xu $S$ \& Zhao Y: Risk factors for posttraumatic stress reactions among Chinese students following exposure to a snowstorm disaster. BMC Public Health 2011; 11:96

Correspondence:

Secil Ozen, $M D$

Turgut Ozal University, Medicine School, Psychiatry Department

06510, Ankara, Turkey

E-mail:drsecilozen@gmail.com 